\title{
État des lieux de l'éducation des filles et des femmes au Mali : contraintes et défis
}

Appraisal of education for girls and women in Mali: Constraints and challenges Estado de la situación en torno a la educación de las niñas y de las mujeres en Malí: limitaciones y retos

\section{Seydou Loua}

\section{CpenEdition} Journals

Édition électronique

URL : https://journals.openedition.org/ries/6571

DOI : 10.4000/ries. 6571

ISSN : 2261-4265

Éditeur

France Education international

Édition imprimée

Date de publication : 1 septembre 2018

Pagination : 103-113

ISBN : 978-2-85420-619-7

ISSN : 1254-4590

Référence électronique

Seydou Loua, «État des lieux de l'éducation des filles et des femmes au Mali : contraintes et défis », Revue internationale d'éducation de Sèvres [En ligne], 78 I septembre 2018, mis en ligne le 01 septembre 2020, consulté le 21 septembre 2021. URL : http://journals.openedition.org/ries/6571 ; DOI : https:// doi.org/10.4000/ries.6571 


\title{
État des lieux de l'éducation des filles et des femmes au Mali : contraintes et défis
}

\author{
Seydou Loua \\ Université des lettres et des sciences humaines \\ de Bamako
}

La situation de la femme dans les pays d'Afrique subsaharienne, des Indépendances à nos jours, a toujours été une préoccupation importante dans les politiques d'éducation et de développement. Tout en reconnaissant les avancées dans sa situation sociale, économique et culturelle, la femme reste encore, dans les pays d'Afrique de l'Ouest, à l'ombre de l'homme et tributaire en grande partie des décisions masculines.

Les femmes maliennes à l'instar des autres femmes de la sous-région ouestafricaine, se trouvent confrontées à la persistance du système patriarcal qui, en confiant l'exercice du pouvoir à l'homme les exclut de la prise de décision et les maintient dans leur rôle d'épouses. Alors qu'elles doivent être au cœur même des changements qui s'opèrent dans les structures sociales et familiales, ainsi que dans le processus de démocratisation économique et politique dont elles sont les premières à subir les conséquences. (Tounkara, 2015)

$\mathrm{Au}$ Mali, comme en Afrique en général, les hommes et les femmes sont caractérisés par des différences au niveau de la scolarisation, de la division du travail à la maison comme au service, de l'exercice du pouvoir coutumier et institutionnel, de la répartition des revenus, du pouvoir de décision, de l'accès aux ressources. Cet article s'efforce à apporter des éléments de réponses aux questions fondamentales suivantes : quelle est la situation éducative des filles et des femmes au Mali ? Quels sont les obstacles liés à leur éducation ? Quelles alternatives sont envisageables pour améliorer leur situation éducative?

\section{ÉTAT DE LA SCOLARISATION DES FILLES$$
\text { ET DE L'ALPHABÉTISATION }
$$$$
\text { DES FEMMES AU MALI }
$$

Avec l'arrivée du colonisateur français dans les années 1800, les pays d'Afrique occidentale française (AOF) ont connu une nouvelle forme d'éducation, à savoir l'école française. Cette école a été d'abord rejetée car les Africains ne s'y reconnaissaient pas. Elle avait peu de rapport avec leurs réalités de terroir et 
utilisait une langue étrangère. Il est important de rappeler que le colonisateur cherchait à imposer sa domination en ne scolarisant que les fils de chefs coutumiers. Le gouverneur général Ernest Roume, gouverneur général de l'AOF de 1902 à 1907, expliquait ainsi les raisons de cette sélection :

Choisissons judicieusement cette minorité, considérons l'instruction comme une chose précieuse qu'on ne distribue qu'à bon escient et limitons-en les bienfaits... Choisissons nos élèves tout d'abord parmi les fils de chefs et de notables car la société indigène est très hiérarchisée. (Moumouni, 1998)

À l'indépendance, en 1960, le taux de scolarisation au Mali était entre 8 et $10 \%$ et l'alphabétisation presque inexistante (Diambomba, 1980), ce qui a conduit le pays à opérer, à partir de 1962, des réformes et innovations structurelles et pédagogiques dans son système éducatif. L'amélioration de la situation éducative de la jeune fille et des femmes était dans l'esprit de toutes ces réformes. La réforme de 1962, avec la démocratisation de l'enseignement, et le programme décennal de développement de l'éducation et de la culture (Prodec), qui a pris fin en 2012, avec son objectif «Un village, une école et/ou un centre d'éducation pour le développement », ont permis d'améliorer la scolarisation des filles.

L'accent sur l'éducation des filles en Afrique, auquel le Mali a pris part, s'est traduit par un certain nombre d'engagements essentiels, dont la Plateforme d'action de Beijing, en 1995, qui était un programme ambitieux pour l'autonomisation des femmes, impliquant des représentants de 189 gouvernements, et le Cadre d'action de Dakar (adopté par le Forum mondial sur l'éducation, en avril 2000) en faveur d'une Éducation pour tous et des objectifs du Millénaire pour le développement. En Afrique, la décennie 2006-2015 a été marquée par des engagements et des objectifs spécifiques, comme l'élimination des disparités liées au genre, afin de garantir l'égalité, l'autonomisation des filles et des femmes par le biais de l'éducation. La biennale de l'Association pour le développement de l'éducation en Afrique (ADEA), en 2008, a conforté cet état de fait, en faisant de la transition de l'éducation de base vers les niveaux supérieurs une priorité et en proposant plus d'enseignants de sexe féminin au niveau post-primaire. Les congrès internationaux des ministres de l'éducation, tel que celui de la Communauté économique des États de l'Afrique de l'Ouest (CEDEAO), en 2004, ont donné la priorité aux engagements pour l'éducation des filles.

Si des améliorations ont été enregistrées dans certains aspects de l'éducation des filles grâce à l'attention que celle-ci suscite dans le monde, il reste beaucoup à faire pour atteindre la parité des genres. Les tableaux qui suivent fournissent quelques statistiques du ministère de l'éducation nationale malienne et de l'Unicef.

Ces tableaux montrent la fréquentation par genre de l'enseignement fondamental, les taux de participation à l'enseignement secondaire et l'évolution des effectifs d'enseignants de l'enseignement secondaire et supérieur malien, de 2014 à 2017. On y remarque que les effectifs des filles au niveau des taux de scolarisation et d'achèvement des cycles, ainsi que ceux des femmes enseignantes 
Tableau $\mathrm{n}^{\circ} 1$

Taux de scolarisation et d'achèvement de l'éducation primaire de 2014 à 2017

\begin{tabular}{|c|c|c|c|c|c|c|c|}
\hline \multicolumn{5}{|c|}{ Premier cycle } & \multicolumn{4}{c|}{ Second cycle } \\
\hline \multicolumn{7}{|c|}{ Scolarisation } & \multicolumn{2}{|c|}{ Achèvement } & \multicolumn{2}{c|}{ Scolarisation } & \multicolumn{2}{c|}{ Achèvement } \\
\hline Garçons & Filles & Garçons & Filles & Garçons & Filles & Garçons & Filles \\
\hline $\mathbf{2 0 1 4 - 2 0 1 5}$ & $63,4 \%$ & $53,8 \%$ & $45,7 \%$ & $52,9 \%$ & $47,8 \%$ & $32 \%$ & $24,2 \%$ \\
\hline $74,8 \%$ & $64,3 \%$ & $49,4 \%$ & $43 \%$ & $55,5 \%$ & $43,7 \%$ & $32,1 \%$ & $23,8 \%$ \\
\hline $\mathbf{2 0 1 5 - 2 0 1 6}$ & & & & \\
\hline $75 \%$ & $\mathbf{7 0 1 6 - 2 0 1 7}$ & & & & \\
\hline $75,8 \%$ & $64,4 \%$ & $48,8 \%$ & $41,3 \%$ & $55,6 \%$ & $46,8 \%$ & $33,2 \%$ & $26,9 \%$ \\
\hline
\end{tabular}

Source : Ministère de l'éducation nationale du Mali, 2018.

Tableau $\mathrm{n}^{\circ} 2$

Taux brut de l'enseignement secondaire et d'alphabétisation

\begin{tabular}{|l|c|c|c|c|}
\hline & \multicolumn{2}{|c|}{ Taux de participation à l'école secondaire } & \multicolumn{2}{c|}{ Taux d'alphabétisation (15-24 ans) } \\
\hline Genre & Filles & Garçons & Femmes & Hommes \\
\hline$\%$ & 25 & 30 & 38,8 & 56 \\
\hline
\end{tabular}

Source : data.unicef.org (2014-2015).

Tableau $\mathbf{n}^{\circ} 3$

Professeurs d'enseignement secondaire (2016-2017)

\begin{tabular}{|l|c|c|c|}
\hline \multirow{2}{*}{ STATUT } & \multicolumn{3}{|c|}{ TOTAL } \\
\cline { 2 - 4 } & Hommes & Femmes & Total \\
\hline Public & 3953 & 900 & 4853 \\
\hline Privé & 16005 & 2395 & 18400 \\
\hline Total & $\mathbf{1 9 9 5 8}$ & $\mathbf{3 2 9 5}$ & $\mathbf{2 3 2 5 3}$ \\
\hline
\end{tabular}

Source : Ministère de l'éducation nationale du Mali, 2018.

Tableau $n^{\circ} 4$

Professeurs d'enseignement supérieur

\begin{tabular}{|l|c|c|c|c|}
\hline Années / Genre & \multicolumn{2}{|c|}{2010} & \multicolumn{2}{c|}{2015} \\
\hline Hommes & 1029 & $92 \%$ & 2173 & $86 \%$ \\
\hline Femmes & 94 & $8 \%$ & 370 & $14 \%$ \\
\hline Total & 1123 & & 2543 & \\
\hline
\end{tabular}

Source : Cellule de planification et de statistique, 2016. 
au niveau secondaire et supérieur sont toujours inférieurs à ceux des élèves garçons et des hommes enseignants. À la faculté des sciences humaines et des sciences de l'éducation de Bamako, qui compte quatre départements d'enseignement et de recherche, la disparité de genre dans le corps enseignant est notoire : le département de sciences de l'éducation compte 23 enseignants permanents, dont une femme, le département de socio-anthropologie en compte 47, dont sept femmes, le département de philosophie en compte neuf, dont deux femmes et le département de psychologie en compte sept, dont une femme. Les filles sont moins scolarisées et connaissent plus de situations de décrochages scolaires que les garçons à tous les niveaux du système. Au niveau de l'alphabétisation, les hommes ont un effectif plus élevé que celui des femmes selon les statistiques de l'Unicef de 2015. Dans les centres d'alphabétisation, comme celui du quartier de Missabougou à Bamako, les métiers comme la menuiserie, la plomberie, la maçonnerie, etc., sont considérés comme réservés aux hommes, les femmes fréquentant le centre pour apprendre la teinture et la couture.

\section{IMPORTANCE DE L’ÉDUCATION DES FILLES ET DES FEMMES}

Selon le rapport de la conférence de Beijing (1995), il est dit que :

Filles et garçons ont tout à gagner d'un enseignement non discriminatoire qui, en fin de compte, contribue à instaurer des relations plus égalitaires entre les femmes et les hommes. Les femmes ne pourront prendre une part plus active au changement que si l'égalité d'accès à l'éducation et l'obtention de qualifications dans ce domaine leur sont assurées. L'alphabétisation des femmes est un important moyen d'améliorer la santé, la nutrition et l'éducation de la famille.

Ann Cotton, de l'ONG Campaign for Female Education, lors d'un entretien accordé au journal Le Monde en 2014, considère que "rien n'a plus d'effets pour une nation que la scolarisation des filles, car une fille éduquée a des revenus $25 \%$ supérieurs à ceux d'une fille analphabète. [...] Elle est plus autonome, se marie et a des enfants plus tard. ${ }^{1}$

L'Unicef a établi que l'éducation des filles permet de réduire la pauvreté, de prévenir les maladies et la violence, de contrôler la natalité et de réduire l'instabilité politique. Selon l'ONG Plan International, en Afrique, les enfants de mères qui ont été scolarisées pendant au moins cinq ans ont $40 \%$ de chances supplémentaires de vivre au-delà de l'âge de cinq ans. La même ONG précise que, selon la Banque mondiale, une augmentation de $1 \%$ du nombre de femmes diplômées de l'enseignement secondaire fait augmenter le revenu annuel par personne de $0,3 \%$. La scolarisation de la fille jusqu'à l'obtention d'un diplôme ou son alphabétisation pour améliorer ses chances de trouver un emploi peut contribuer au bien-être de la famille.

1. Voir : [lemde.fr/2AnriMp/]. 
L'écart entre les filles et les garçons se creuse à l'école à mesure que ceux-ci progressent dans l'enseignement car les filles ont moins tendance à achever leur cycle primaire que les garçons. Selon l'ONG Plan international, dans un rapport de 2012, dans 47 pays africains, les filles ont réellement moins de $50 \%$ de chance d'achever le cycle primaire. Dans un rapport publié en 2015, l'ONU estimait que 57 millions d'enfants n'étaient pas scolarisés dans le monde, dont 33 millions en Afrique subsaharienne, avec une proportion de $55 \%$ de filles. En abondant dans le même sens que l'ONU, dans le rapport du Programme d'analyse des systèmes éducatifs de la Confemen (Pasec, 2015), on remarque que lorsque les différences liées au genre existent au niveau de l'école, elles sont toujours en faveur des garçons.

\section{ObStACLes à la scolarisation DES FILLES AU MALI}

$\mathrm{Au}$ Mali, les filles sont confrontées à certains obstacles en ce qui concerne l'accès à l'école, le maintien dans le système scolaire, l'orientation et la qualité des enseignements reçus.

\section{L'obstacle des coûts financiers}

L'école n'est pas totalement gratuite, car les familles prennent en charge les frais d'inscription, de fournitures, de déplacements, etc. Plus l'élève avance dans sa scolarité, plus ces frais augmentent et moins la fille a de chance de poursuivre ses études car les familles modestes préfèrent investir dans l'éducation du garçon, lequel est considéré de fait comme étant la relève du chef de famille. La dot, dont les montants diffèrent selon les familles, peut motiver les parents à « donner la fille en mariage » assez tôt. Selon l'Unicef (2001), « quand la misère est grande, une fille jeune peut représenter une charge, et son mariage est une stratégie de survie familiale dans les sociétés traditionnelles d'Afrique subsaharienne ».

\section{La violence faite aux filles}

La violence ainsi que la peur de la violence dans et hors des écoles sont des entraves à la scolarisation et à l'achèvement du cursus pour les filles. Des violences de la part des enseignants ou d'autres personnels, notamment le harcèlement sexuel ou les discriminations de genre, ne sont pas rares. Lange (1998), parlant de la scolarisation des filles en Afrique subsaharienne, note que "la violence verbale et souvent physique dont sont victimes les filles scolarisées indique à quel point la scolarisation des filles peut paraître comme une agression auprès de certains groupes ».

\section{Les distances à parcourir}

L'éloignement de l'école peut constituer une entrave à la scolarisation et au maintien de la fille à l'école dans le milieu rural. Lorsque l'école est trop éloignée des enfants, les parents préfèrent déscolariser les filles, qui, en raison 
des tâches ménagères qu'elles ont à effectuer, ne peuvent pas supporter les distances quotidiennes à parcourir. L'alternative de la famille d'accueil dans le village où est située l'école est souvent envisagée, mais les difficultés rencontrées dans les familles d'accueil conduisent certaines filles à abandonner l'école.

\section{Les facteurs pédagogiques}

Les enseignants peuvent avoir des préjugés sexistes, de manière consciente ou inconsciente. Dans les rapports élèves-enseignants, ils n'ont pas la même attitude vis-à-vis des garçons et des filles, ce qui peut décourager les filles ou limiter leurs performances. Certains manuels scolaires peuvent donner une image négative de la fille, en mettant en avant une supposée supériorité de l'homme. Dans la collection de livres de lecture Mamadou et Binéta éditée en France par Edicef et utilisée par l'école malienne, les personnages masculins sont toujours plus nombreux et ont de meilleurs rôles que les personnages féminins. Selon l'Unicef (2013), en 2012, seules $12 \%$ des écoles disposaient de latrines adaptées aux filles.

\section{Le mariage et la grossesse précoces}

Selon Plan international, en Afrique, plus de $50 \%$ de filles donnent naissance à leur premier enfant avant l'âge de 21 ans. La grossesse précoce entraîne souvent un mariage précoce, pour préserver l'honneur de la famille dans un milieu où la population reste profondément ancrée dans sa culture. Au Mali, entre 2006 et 2011, 50,4\% des mariages concernaient les filles âgées de 15 à 19 ans et $20 \%$ de ces filles vivaient dans des familles polygames (Walker, 2013). Selon l'Enquête démographique et de santé au Mali (2012-2013), une femme sur cinq (20\%) était déjà en union avant d'atteindre l'âge de 15 ans. La moitié des femmes (50\%) était déjà en union avant 18 ans. Cependant, l'âge moyen au moment du premier mariage est passé de 13-15 ans pour les filles à 25-26 ans, tandis que celui des jeunes garçons est passé de 20 ans à près de 35 ans. La même source indique que les femmes qui n'ont aucun niveau d'instruction ont, en moyenne, 2,5 enfants de plus que celles qui ont un niveau secondaire ou plus; près de deux adolescentes sur cinq (39\%) ont déjà commencé leur vie reproductive et $33 \%$ ont eu, au moins, un enfant. La jeune fille mariée connaît donc un décrochage scolaire plus précoce, avec le poids des activités familiales.

\section{Le poids de la tradition et des activités familiales}

Avant l'introduction de l'islam au VIII siècle et l'arrivée des Français au Mali vers 1820 (Stern, 2009), tous les groupes sociaux disposaient d'un système éducatif qui permettait de donner à l'enfant une éducation et une formation afin 
de l'intégrer progressivement dans son milieu de vie. Dans l'éducation traditionnelle, les garçons et les filles ne subissent pas les mêmes influences et les rôles sont partagés : pendant que le garçon suit son père dans des activités considérées comme étant masculines, la fille suit sa mère dans ses activités dites féminines. Chacun des deux sexes participe au développement et à la cohésion de la société de différentes manières. L'homme étant d'emblée chef de famille, il est chargé de la recherche du gain quotidien, tandis que la femme est appelée à s'occuper des activités au sein de la famille relevant du ménage et de l'entretien des enfants. La reproduction sociale du rôle de future épouse de la fille commence à l'adolescence, bien qu'elle soit scolarisée. La fréquence des travaux domestiques a donc un impact sur le rendement scolaire des filles, ce qui se manifeste par de l'inattention en classe, de l'absentéisme, de la fatigue, des retards, etc.

En matière d'éducation, les filles sont toujours confrontées à la discrimination dans bien des régions du monde, du fait des traditions, des mariages et grossesses précoces, de la pénurie d'établissements scolaires convenablement équipés et d'accès facile. On attend des jeunes filles qu'elles s'acquittent de leurs obligations scolaires sans négliger leurs tâches domestiques, ce qui se traduit par des résultats scolaires médiocres et des abandons précoces. (Rapport de la conférence de Beijing, 1995)

Ce passage de la conférence qui correspond à la réalité malienne et que nous pouvons vérifier à travers nos observations et différentes théories sur la question, est cependant à relativiser, car il correspond à une vision plus citadine de la question. Dans le milieu rural malien, où vit environ $74 \%$ de la population, les garçons ont aussi des occupations extrascolaires, notamment dans les champs et les pâturages. L'accès à l'éducation est problématique pour les deux sexes dans le milieu rural, même si les filles connaissent plus de décrochages que les garçons car ces derniers ne sont pas confrontés à certains obstacles mentionnés ci-dessus.

\section{Les considérations religieuses}

Avec l'avènement de l'islam, les pratiques coutumières se sont légitimées à travers les interprétations parfois fallacieuses des préceptes islamiques. Traditionnellement et dans une certaine conception de l'islam, qui est la principale religion au Mali, la femme doit se marier tôt, procréer de nombreuses fois, évoluer dans la sphère privée. Dans ces conditions, envoyer une fille à l'école n'a que peu de sens pour les familles, puisque ce qu'elle y apprendra ne lui servira pas pour son rôle d'épouse. Les écoles coraniques et les médersas sont privilégiées pour les filles par certaines familles, au nom de l'islam. La femme, dans les sociétés arabes avant l'arrivée de l'islam, appartenait à son mari et n'avait pas droit à l'héritage, comme c'est le cas dans la société traditionnelle malienne. Comme dans de nombreuses sociétés traditionnelles, le statut de la femme est inférieur à celui de l'homme. L'arrivée d'une religion comme l'islam peut 
perpétuer cet état de fait, même si une lecture éclairée du texte religieux permet aussi une amélioration de la situation des femmes, comme par exemple le verset 19, sourate $4 \mathrm{du}$ Coran : "Il ne vous est pas licite d'hériter des femmes contre leur gré...». Il est donc évident que de telles pratiques coutumières légitimées au nom de la religion musulmane émanent de la volonté des hommes d'imposer des traitements arbitraires et des classifications sans fondement juridique, qui ne répondent en réalité qu'à la satisfaction du sexe masculin, lequel cherche à tout prix à pérenniser sa mainmise sur la femme.

\section{L’insécurité}

Dans certaines régions du nord et quelques zones du centre du pays, l'insécurité, liée au terrorisme que le Mali connaît depuis 2012, constitue aussi un obstacle à la scolarisation. Le fait que ceux qui se nomment djihadistes ou islamistes, comme le Front de libération du Macina, s’insurgent contre l'école occidentale, entrave la scolarisation des filles. Cette situation entraîne la fermeture des écoles et le bouleversement de l'éducation dans les régions concernées. Les filles sont particulièrement touchées par ces formes de terrorisme, car elles sont victimes d'enlèvements et de viols. Dans un rapport d'enquête de la Coordination nationale de lutte contre la prolifération des armes légères, le secrétaire à l'information du conseil communal des jeunes de Mopti (cinquième région au centre du Mali) affirme qu'en 2013, 436 écoles ont été fermées à Mopti, avec environ 18000 enfants privés d'école. En juin 2017, l'Unicef a indiqué que 194 écoles ont été fermées dans la région de Mopti, avec plus de 80000 enfants désormais non scolarisés et que le conflit a mis en péril la scolarité de 200000 enfants. Selon la Banque mondiale, 153 écoles sont encore fermées dans les régions de Gao, Tombouctou et Kidal, en 2018.

\section{Alternatives d'amélioration DE L'ÉDUCATION DES FILLES ET DES FEMMES AU MALI}

Les pesanteurs traditionnelles et religieuses sur l'éducation des filles pourront être contrées à travers la sensibilisation des populations rurales au sujet de l'importance de l'école pour le genre féminin, la lutte, par l'implication des leaders religieux, contre l'usage discriminatoire de textes considérés comme étant sacrés et des mesures administratives visant à aboutir à une amélioration de la situation éducative de la fille.

Le développement et le renforcement de la législation nationale sur l'interdiction du mariage des enfants peuvent être des mesures dissuadant les parents de «donner leurs filles en mariage » avant la fin de l'enseignement fondamental, qui dure neuf ans. L'intensification des campagnes de sensibilisation pour éveiller les consciences sur les conséquences du mariage des enfants et des grossesses précoces, par l'implication des chefs religieux et coutumiers, des 
hommes et des garçons, peut être efficace dans les milieux ruraux pour favoriser l'éducation des filles. Certaines organisations non gouvernementales, notamment les ONG Agir et Save the children, travaillent déjà dans le sens du renforcement de l'éducation à la sexualité dans des écoles, pour assurer l'accès aux services de santé et réduire les grossesses précoces des élèves dans la région de Sikasso (troisième région du Mali). Ces organisations ont impliqué dans leur programme les grand-mères (qui sont, au Mali, sont les confidentes des jeunes filles), les agents de santé, les associations de jeunesses villageoises et les enseignants. L'État malien, à travers les services d'action sociale et les partenaires de l'école, devra accorder des aides financières aux familles démunies pour scolariser et maintenir les filles à l'école durant la période de scolarité obligatoire. En plus de la redynamisation de l'axe référentiel du Prodec «Un village, une école », les mairies, les partenaires, les comités de gestion scolaire devront se fixer comme objectif la mise en place du cycle complet de l'enseignement fondamental dans les villages, afin d'éviter les changements de localité qui sont préjudiciables aux filles et qui constituent des motifs d'abandon scolaire. Au regard de témoignages recueillis dans la région de Sikasso, les auteurs des grossesses des écolières sont souvent des membres de la famille d'accueil. La prise en compte de la question du genre dans le recrutement du personnel enseignant, par l'augmentation du nombre de femmes, peut limiter l'inégalité de genre dans l'enseignement.

Le Burkina Faso a expérimenté certaines stratégies pouvant être mises à l'essai au Mali, comme l'assouplissement du temps d'enseignement, avec des horaires souples pour les filles qui ont trop d'activités domestiques et n'arrivent pas à suivre les cours au rythme normal. Les autorités scolaires organisent des cours de renforcement scolaire, dispensés à ces filles pour les mettre au même niveau que leurs camarades garçons.

\section{Mesures en faveur de l'éducation des filles et des femmes au Mali}

Les autorités politiques et scolaires maliennes, conscientes de la situation, ont été à l'origine de mesures favorisant la situation éducative des filles et des femmes.

En dehors du programme Éducation pour tous, piloté par le ministère de l'éducation nationale, d'autres mesures spécifiques ont été prises pour favoriser l'éducation des filles. La mise en place, en 1992, de la Cellule nationale de scolarisation des filles et de ses antennes dans huit régions à l'époque (dix aujourd'hui), avait pour but de tenir compte des spécificités régionales et locales et de favoriser une meilleure implication des partenaires de l'école dans la promotion de la scolarisation des filles. Il a été mis en place des réformes comme la "Nouvelle école fondamentale» en 1994 et le Prodec, en 1998, avec des objectifs d'amélioration de la situation scolaire de la jeune fille. Il y a eu en 
2000 la création de la Section de la scolarisation et formation des filles et la mise en place depuis 2011 de la Division de la scolarisation des filles, pour la promotion de la scolarisation et du maintien des filles à l'école. La Politique nationale genre, de 2011, et le Fonds d'appui à l'autonomisation de la femme et à l'épanouissement de l'enfant, en 2012, ont été initiés pour améliorer l'insertion professionnelle des femmes. La mise en ouvre de la politique nationale de l'alimentation scolaire, à travers des cantines dans les écoles fondamentales situées à plus de cinq kilomètres des domiciles, soutenue par les mairies, les familles et les partenaires, est jugée efficace et elle est toujours en cours pour maintenir les filles à l'école. Il a été mis en place une politique d'encouragement des activités génératrices de revenus, comme le maraîchage, la couture, etc., à l'adresse des femmes pour réduire le travail des enfants et favoriser le maintien des filles à l'école.

Pour l'égalité entre les sexes et l'autonomisation des femmes, les objectifs du Millénaire pour le développement ont voulu éliminer avant 2005 les disparités de genre dans l'enseignement primaire et secondaire et avant 2015 à tous les niveaux de l'éducation ainsi que réduire les disparités entre les sexes dans la vie économique et politique du Mali. La Politique nationale genre du Mali, qui est en vigueur depuis 2013, promeut une démarche inclusive qui concerne toutes les composantes de la société dans toutes les régions; une approche systémique de l'intégration de l'égalité femmes/hommes dans les réformes publiques et les institutions du pays, qui cible les secteurs les plus porteurs d'égalité et dont les résultats pourront être perceptibles par la population; l'amélioration de l'accès égal à l'éducation et à la formation ; la promotion de l'équilibre dans la représentation des femmes et des hommes dans les instances de décision et dans les postes électifs et administratifs au niveau national et international.

Bien que ces mesures soient salutaires, la situation éducative des filles et femmes reste insatisfaisante au Mali. Selon Tounkara (2018), la promotion de la femme est souhaitée par les autorités politiques, mais entre le principe et la réalité, il y a un fossé difficile à franchir, car la discrimination à l'égard des femmes reste un fait difficile à nier. Maintenant que tous les droits des femmes ont été institutionnellement reconnus, il s'agit à présent de réaliser leur promotion concrète en mettant un accent particulier sur la scolarisation des filles. Beaucoup de filles abandonnent l'école de façon prématurée, trop de femmes restent non lettrées et peu d'entre elles peuvent accéder à des postes de responsabilité. Selon l'Institut national de la statistique du Mali, en 2014, le pays comptait quatorze femmes députées sur 147, huit femmes maires sur 703, trois femmes ambassadrices sur 36, etc. Le Mali prend donc des engagements régionaux et internationaux pour l'égalité de genre, mais connaît beaucoup d'insuffisances au niveau de l'instauration de l'équité entre homme et femme. 


\section{BibLIOGRAPHIE}

Institut national de la statistique, cellule de planification et de statistique (2012): Enquête démographique et de santé au Mali, 2012-2013, Gouvernement du Mali/ USAID.

Institut national de la statistique du Mali, Gouvernement du Mali (2014) : Enquête modulaire et permanente auprès des ménages EMOP, Rapport d'analyse premier passage 2014, Institut national de la statistique du Mali (INSTAT).

DIAMBOMBA M. (1980): La réforme scolaire au Mali: Essai d'analyse des facteurs qui atténuent ses résultats, Québec, Université Laval/Faculté des sciences de l'éducation.

LANGE M.-F. (1998) : L'école et les filles en Afrique, scolarisation sous conditions. Paris, Karthala.

MOUMOUNI A. (1998): L'éducation en Afrique, Paris, Dakar, Éd. Présence africaine.

ONU (1995) : Déclaration et programme d'action de Beijing, ONU Femme. En ligne [https://goo.gl/fCfmeb].

ONU (2015) : Rapport sur les Objectifs du Millénaire pour le Développement, New York. En ligne [https://goo.gl/MLJ1Ta].

OPHEIM M. (2000) : «Les filles et l'école au Mali », Nordic Journal of African Studies, p. 152-171. En ligne [https://goo.gl/pGfC5v].

Plan international (2012): Parce que je suis une fille, rapport Afrique 2012, Plan/ Bureau régional Afrique de l'Ouest.

Programme d'analyse des systèmes éducatifs de la CONFEMEN (2015) : Performance des systèmes éducatifs en Afrique subsaharienne francophone, Dakar, PASEC.

STERN L. (2009) : L'école au Mali, hier et aujourd'hui, Gymnase Auguste Piccard.

TOUNKARA A. (2018) : "Des pratiques culturelles à l'islamisation du statut de la femme au Mali », Revue Recherches Africaines/Université des lettres et des sciences humaines de Bamako, $\mathrm{n}^{\circ}$ 18-19, janvier, p. 273-283.

TOUNKARA A. (2015) : Femmes et discriminations au Mali, Paris/Harmattan (Études africaines).

UNESCO (2015) : Examen national 2015 de l'Éducation pour tous : Mali, UNESCO. En ligne [https://goo.gl/cCgKgv].

Unicef (2001) : Le mariage précoce, Florence : Centre de recherche Innocenti.

Unicef (2013): Situation des femmes et des enfants au Mali. En ligne [https:uni. cf/2LDW9cj].

WALKER A.-J. (2013) : Mapping Early Marriage In West Africa. A scan of trends, interventions, what works, best practices and the way forward, The Ford Foundation, West Africa Office. En ligne: [https://goo.gl/hPiW4n]. 
